\title{
Problems and countermeasures in the restoration project of cultural relics of Macao Chong Sai Pharmacy
}

\author{
Liang Zheng ${ }^{1, *}$ and Yile Chen ${ }^{1}$ \\ ${ }^{1}$ Macau University of Science and Technology, Avenida Wai Long Taipa Macau, China
}

\begin{abstract}
The Macau Chong Sai Pharmacy has a long history and rich historical materials. It is not only a cultural relic of Sun Yat-sen's old democratic revolution in Macau, but also the former residence of the new democratic general He Chang in Macau. It is also the site of the SinoWestern maritime trade transit terminal. Triple cultural relic value.
\end{abstract}

\section{Introduction}

\subsection{Historical background}

The Macau Chong Sai Pharmacy has a long history and rich historical materials. It is not only a cultural relic of Sun Yat-sen's old democratic revolution in Macau, but also the former residence of the new democratic general He Chang in Macau. It is also the site of the SinoWestern maritime trade transit terminal. Triple cultural relic value [1].

After excavating the underground of the Chong Sai Pharmacy, archaeological discoveries of large-scale granite stone structure wharves and unearthed Clark porcelain tiles that were mainly for export in the late Ming and early Qing dynasties, and compared historical materials, maps, coastline relics and stratigraphic exploration, inferred here In the period, before the establishment of the Chong Sai Pharmacy, and even at the beginning of the land reclamation of Caodui Street, it was a transit terminal for Chinese and Western freight along the coast of Beiwan, Macau, and also provided archaeological evidence for Macao as an important Chinese and Western freight port during the Ming and Qing Dynasties [2].

Sun Yat-sen graduated from the Hong Kong Academy of Western Medicine in 1892. During his studies, he was invited to help the Macau gentry in diagnosis and treatment. He established a good reputation in Macau because of his exquisite medical skills and cure all diseases. In the same year, he went to Macau Jinghu Hospital to preside over the first western medicine and western medicine work. On December 18th, he was funded by the Macau Gentry and Jinghu Hospital to open the Chong Sai Pharmacy. Cao You, one of the founders of Jinghu Hospital, rented No. 80 Caodui Street to Sun Yat-sen. As a place of Chong Sai Pharmacy [3]. Sun Yat-sen met a large number of Chinese and Portuguese people in the upper class of Macau during his medical practice at the Chong Sai Pharmacy, and established a

\footnotetext{
* Corresoinding author: zlanger@163.com
} 
deep friendship and trust, which was an important help for the subsequent development of the revolutionary cause.

On September 25, 1893, Sun Yat-sen opened the East-West Pharmacy and the Chong Sai Pharmacy in Guangzhou and Zhongshan before Sun Yat-sen, and the Macao Chong Sai Pharmacy was commissioned by a friend [4]. After the death of Cao You in 1896, the Chong Sai Pharmacy was handed over to his son Cao Shanye. The Bureau of Traditional Chinese Medicine remained in operation for many years as one of the bases to cover the revolutionary party and their activities in Macau [5].

Check the information of the Macau Property Registration Bureau. On February 29, 1908, it was closed for auction by the Portuguese government. In 1947, He Shi sold it in Chen Shi, during which it was changed into shops such as Butterfly Horse Shop, Wanxin Horse Shop and Guangyu Electric Appliance Store. After the return of Macau, it has been vacant until it was purchased by the Macau SAR government and repaired in 2011. Work [6].

Although Sun Yat-sen has participated in the operation of the Macau Chong Sai Pharmacy for less than a year, it is an important place for his youth to start a business, an industrial park for the development of western medicines and a base for revolutionary movements. According to the record of the "London Menace" written by Sun Yat-sen, the Macau Chong Sai Pharmacy It is also the first Chinese western medicine museum in China[7]. Therefore, the Chong Sai Pharmacy is the most historical and culturally significant cultural relic building among Sun Yat-sen's relics in Macau.

\section{Building features}

The Chong Sai Pharmacy is located at No. 80 Caodui Street, Macau (Picture 1), with a width of about 5 meters, a depth of about 28 meters, and a height of about 17.5 meters. It is divided into three floors. It is a typical Lingnan bamboo tube house building with the layout of the lower story and upper story (pictured 2). The building is a brick-wood structure, with hard mountain purlins, double-slope tile roofs, and bright light tiles on the roof to enhance the natural lighting in the interior. The wall is made of blue brick walls, with 5 straight and 1 dicing methods, and thin brick joints. The front elevation has a concave door design, with front cornices and gable heads. The door consists of a sliding door and a panel door. The panel door is changed to a glass door after restoration. There are two types of windows: shutters and oyster shell windows. Oyster shell windows are traditionally crafted windows in which the shell is polished into a semi-permeable shell and embed ded in the wooden window frame. It has the function of heat insulation and wind shelter. 


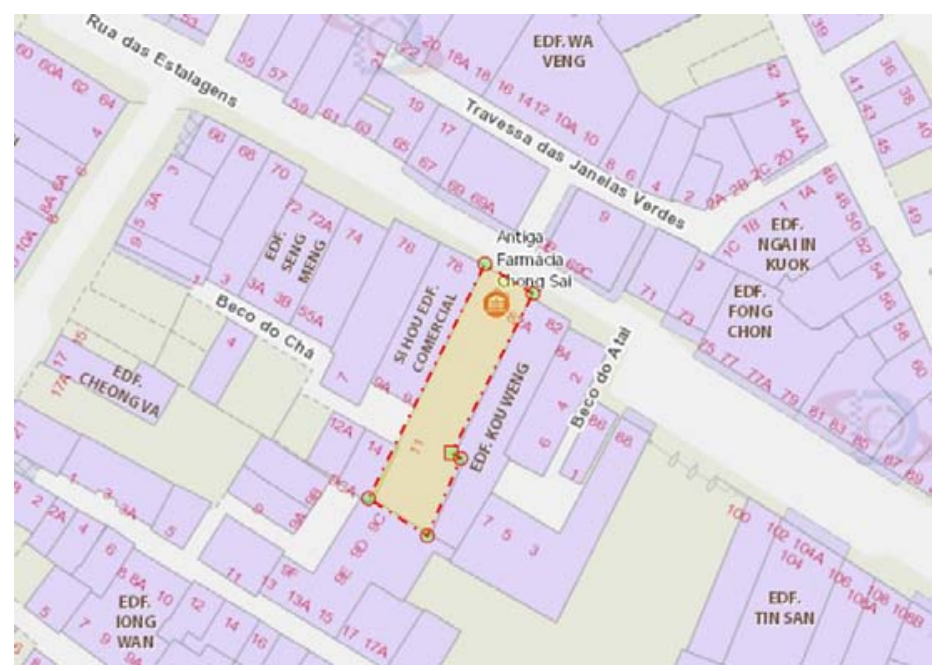

Fig. 1. The floor plan of the Chong Sai Pharmacy (webmap.gis.gov.mo).

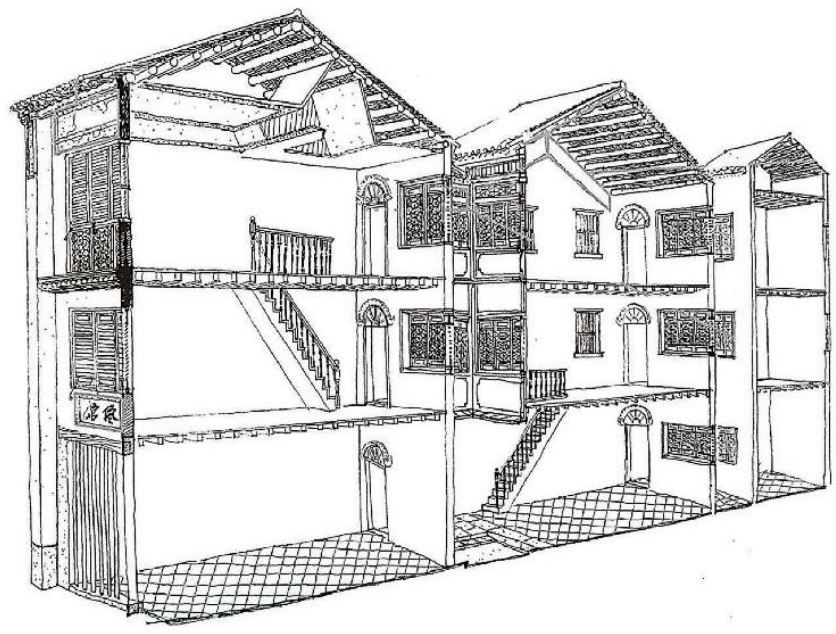

Fig. 2. Sectional view of Chong Sai Pharmacy (Ta Kung Pao [8]).

The facade is three-in-depth, the floor is covered with orange clay stairs, and there are 2 patios in the middle for ventilation and lighting. The middle of the first floor is the relic of the dock stone structure during the Ming and Qing Dynasties. The interior door is an arch, and the lintel is covered with petals. The semi-circular gray clay sculpture, with bat-rat brackets at both ends of the door lintel. The suspended ceiling is square and concave, surrounded by money patterns and flower patterns, which has the effect of breathable and anti-termite. There is an ancient well on the left side of the second entrance on the first floor, and the second entrance on the second floor is on the right. There are two sets of casement windows on the second floor, one enters the wooden ladder, and one enters the third floor. The windows along the street on the third floor are two sets of casement windows. The upper set is shutters with horizontal wooden lattices, and the lower set is oyster shell windows with vertical wooden lattices. On the wall at the second entrance of the building, there is a peach blossom ash clay sculpture from stone. 


\section{The problem of engineering}

Macau is the first stop for Sun Yat-sen to enter society from university, and also the starting point of life and career. He was the first Chinese to use Western medicine to treat patients. The Chong Sai Pharmacy, as an empirical evidence of this experience, has value worth protecting and revitalizing. In 2011, the Macao Special Administrative Region government purchased the building for 36 million patacas and carried out a series of restoration work. Including the restoration of the building's roof, walls and slabs (Figure 3), re-planning the internal space, and installing air-conditioning, elevators, security and fire-fighting equipment for the cultural relics to meet the needs of modern people. In 2012, during the space renovation project, the underground port remains stone structure was excavated, so the internal space layout was further adjusted until the overall building restoration work was completed in December 2016 and it was officially opened to the public.

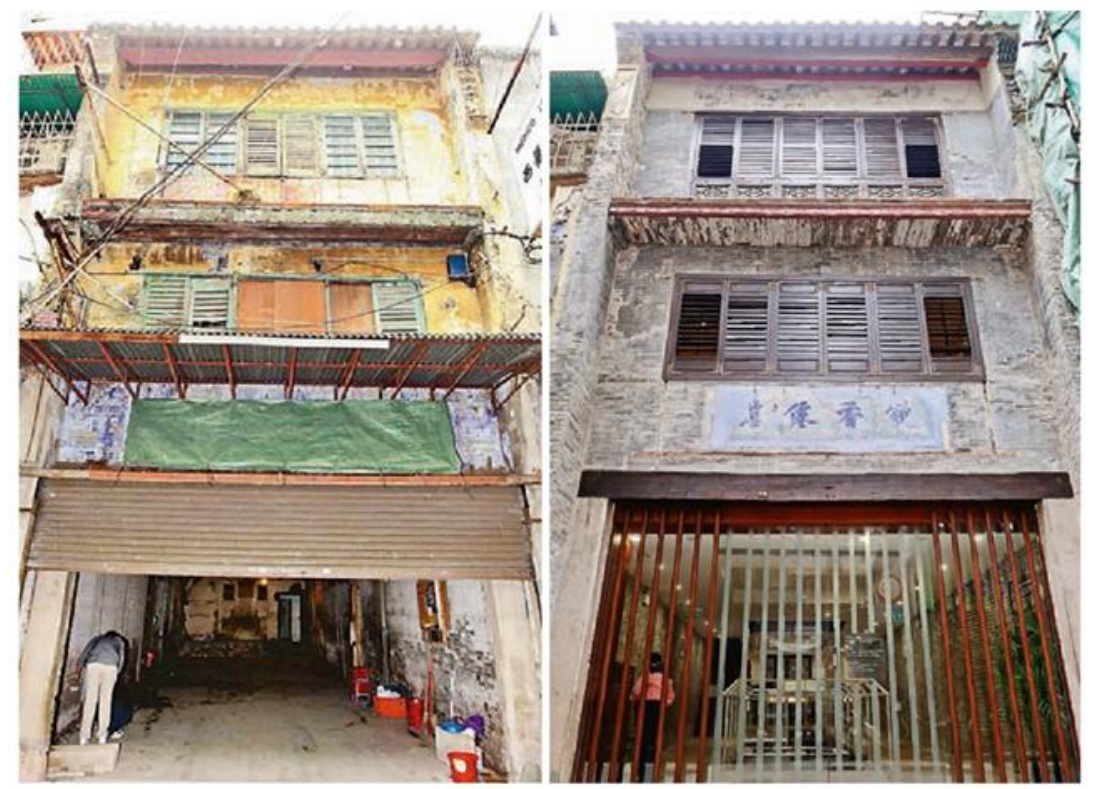

Fig. 3. Comparison of Chong Sai Pharmacy before and after restoration (left old and right new).

The Chong Sai Pharmacy preserve the original structural characteristics as a whole. After restoration, the entrance lobby on the first floor retains the archaeological relics for display, and the excavated cultural relics are placed at the end, including the Clark porcelain and pad porcelain cakes exclusively for export during the Ming and Qing Dynasties. The name of the Clark porcelain is taken from the sailing ship used for trade in Portugal. Carrac", transliterated as "Clark". In addition to porcelain, there are coins, Anping pots, jade cigarette holders and other commodities at the time. The signboard on the facade of the Chinese Medicine Bureau reads "Yinxiang Tiongguan". Combined with the jade cigarette holders found in archaeology, it is speculated that the Chong Sai Pharmacy once sold opium. Cigarette shop. On the second floor is the Sun Yat-sen Exhibition Hall and Library, where there is an installation of "The Voice of Father and Son", where you can listen to the recordings of Sun Yat-sen's past speeches. The third floor mainly displays the design style and materials of the building, including shutters, oyster shell windows, gray plastic and other objects and historical photos. The restored Chong Sai Pharmacy not only allows people to trace Sun Yat-sen's footprints in Macau, but also to feel his patriotism and sentiments of his family and the world. Although the restoration work of the Bureau of Traditional Chinese Medicine has been completed, 
some problems that cannot be ignored have been revealed after the restoration, which is worthy of further study and improvement.

\subsection{The brick gap is too large}

The indoor floor of the Chong Sai Pharmacy is made of orange clay step bricks. The joints of the bricks are about $1 \mathrm{~cm}$. This not only destroys the beauty of the floor, but also causes cracks if the joints are too thick. Especially as the floor tiles, it is easy to cause cracks after the joints are cracked. The tiles are worn or fall off, as shown in Figure 4, the edges of the step tiles on the right side have been worn and turned white. In addition, some floor tiles have been blackened and faded. The brick surface has uneven color, rough texture and lack of daily maintenance. It should be cleaned and maintained in time, otherwise it will easily cause broken windows and reduce the overall environmental quality. The edge of the paving is too hard, the brick body is cut and placed on the edge, and the material is not transitioned. The edge of the brick body after the cut will have uneven gaps, which are easy to seep water, resulting in mildew on the ground and deterioration of the quality of the paving, causing a chain reaction.

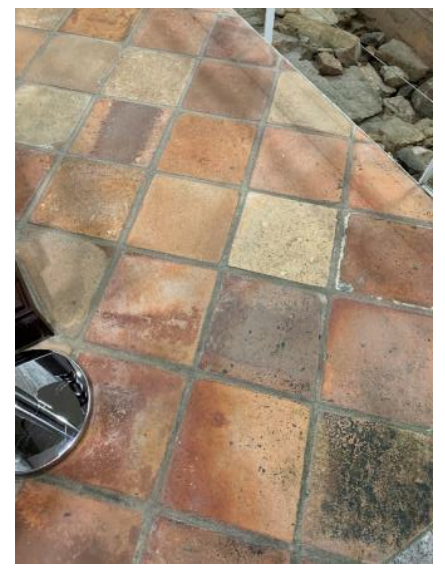

Fig. 4. Ground paving of Chong Sai Pharmacy.

\subsection{Mold on the ceiling}

Water leakage and mildew occurred on the ceiling of the Chong Sai Pharmacy (Figure 5). The reason for this phenomenon is speculated. First, the air-conditioning drainage pipe in the ceiling is leaking, and the ceiling lacks drainage structure, which causes water to accumulate in the potholes, causing mold and water leakage. The phenomenon. Second, because the air conditioner is turned on for a long time and lacks dehumidification equipment, the outdoor air temperature is not balanced with the indoor air temperature, and the air humidity is high, causing condensation water to accumulate on the ceiling, resulting in moldy dripping. Regardless of the reason, it should be treated and prevented in time. Mold can cause air quality degradation and affect human health. Secondly, the ceiling is not easy to clean. In the long term, it will expand the moldy area. The mold will spread in closed rooms and cause other cultural relics in the room. Mildew, resulting in loss of value of cultural relics. 


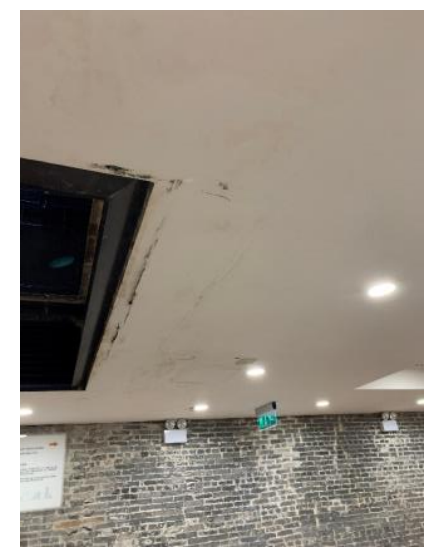

Fig. 5. Ceiling of Chong Sai Pharmacy.

\subsection{Wrong patching of bricks on the wall}

The blue brick wall in the Chong Sai Pharmacy was damaged due to weathering (Figure 6). After the damage, dark yellow salty bricks were used to fill. Not only the appearance is damaged, but the salty bricks are acidic. The hardness and life of the bricks will be greatly reduced, which is easy to cause A breakage of a brick wall. The combination of salty bricks and weakly alkaline blue bricks will affect the surrounding blue bricks, acidify the surrounding bricks, and reduce the overall quality of the wall. From the perspective of cultural customs, salty bricks are often used in places such as cooking fume kitchens, toilets, huts, pig pens and cattle pens, and are not suitable for use in residential interiors, which violates the residents' habits. In addition, the early blue bricks were mostly made by handmoulding, and there would be a difference between concave and convex surfaces. They should be arranged according to the convex surface on the top and the concave surface on the bottom. It was found on the site that some blue bricks were backfilled without paying attention to the unevenness of the blue bricks. Reverse backfilling not only decreased the aesthetics, but also made the stability of the brick wall down. Seriously, it would also cause the brick wall to collapse.

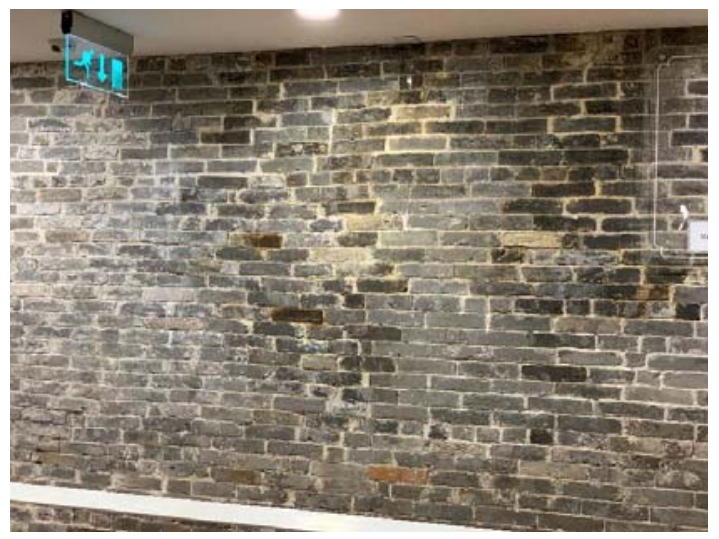

Fig. 6. Brick wall of Chong Sai Pharmacy. 


\subsection{Cement repair wall}

The brick walls of the Chong Sai Pharmacy use cement to fill the gaps and cracks (Figure 7). The physical properties of cement and blue bricks are different. The cement is too hard and impermeable, which will easily cause the brick walls to crack and break again. In addition, the overall color of the brick wall is different from that of the cement. The use of cement to fill the pit affects the beauty of the brick wall, which makes the brick wall less clean and poor in quality. Brick walls have a relatively large proportion of the indoor visual area. Large areas of untidy brick walls will reduce the public's experience of viewing the exhibition and affect the positive effects of cultural communication in the exhibition hall. On the contrary, the public will form negative emotions. In addition, the filling of brick joints overflowing between the blue bricks should be treated in time to keep the brick walls tidy and beautiful to avoid messy caulking.

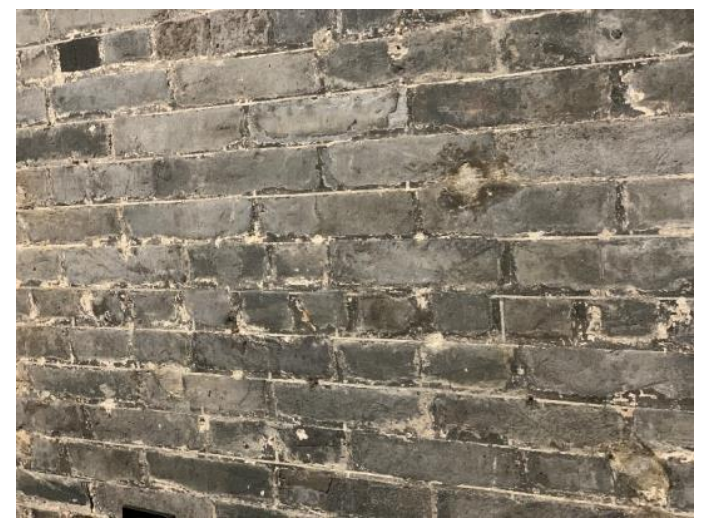

Fig. 7. Detail of brick wall of Chong Sai Pharmacy.

\subsection{Desalting by injection and destroying the wall}

The weathering of the brick walls of Macao's heritage buildings is mainly due to the rise of groundwater and the destruction of crystalline salt on the walls. As Macau is surrounded by sea on all sides, the groundwater source is abundant, and the land is built by the method of dike filling, which makes the bottom of the soil high in salinity. Since the Chong Sai Pharmacy is located at the edge of the original coastline, the groundwater can rise to the wall through capillary action. The wall is dampened and weathered and bulged and collapsed, greatly affecting the life and quality of the building. In addition, the on-site sampling also found that there are nitrate elements on the surface of the brick body. The presence of nitrate proves that the brick body contains a certain amount of salt. Therefore, the brick wall needs to be desalted to protect the integrity of the brick wall and prolong its service life. .

The brick walls of the Chong Sai Pharmacy use the injection method for water interception and desalination projects (Figure 8). This method is not suitable for the double corner walls of Lingnan buildings. The double corner walls of Lingnan buildings are hollow walls, which have the effect of thermal insulation. Injection needs to make holes in the wall to destroy the stable structure of the wall. The principle of injection is to inject water-cutting liquid under the wall, but the effectiveness of the interception cannot be guaranteed. The brick surface of the bottom layer is obliquely cut and the hole is injected to remove salt. It is to be demonstrated, and the picture style used in the schematic diagram displayed on the spot also has errata, and it is not the structural diagram of the double corner wall. In addition, there is an experimental nature of injection desalination. The usual practice is not to use precious cultural relics as experimental objects, but to choose similar old houses for experiments and 
directly experiment on cultural relics, which will cause the risk of destroying cultural relics. After the cultural relics are destroyed, the original stable physical structure is difficult to restore, and the value and beauty of the cultural relics will be further lost.

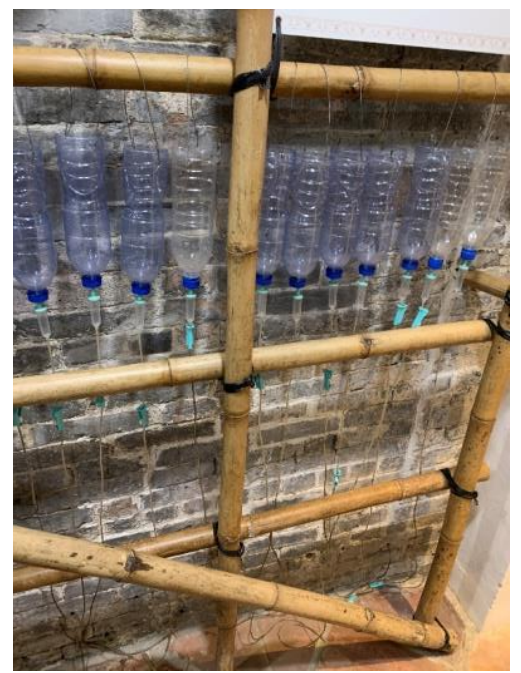

Fig. 8. Desalting by injection.

\section{Responses}

The problems revealed in the restoration project of the Chong Sai Pharmacy cultural relics should be repaired in a more scientific way, respect local customs and historical authenticity, and carefully use restoration engineering techniques to avoid further damage to the cultural relics. In response to the restoration of cultural relics found in the above-mentioned research, the following measures have been taken to restore.

\subsection{Gap tiles}

The caulking materials of the step bricks should be consistent with the original materials, such as lime mortar, etc., avoid using modern materials such as cement, etc., which will lead to the "inconsistent" of the new and old building materials, and the construction techniques and materials are also historical information Part of this should be fully respected, preserved intact, repaired as before, and never "superfluous". Avoid brick joints that are too large, usually about $2 \mathrm{~mm}$. If there is overflow of joint filling materials during the bricklaying process, it should be dealt with in time. After the bricklaying is completed, the excess caulking materials should be manually cleaned up with sanding paper to avoid damage to the beauty of the paving. Finally, the jointing process is used to enhance the cleanliness. Avoid using machines to polish the brick body. There are water barrier and breathable layer on the surface of the old brick. Polishing the brick body will make the brick lose the water barrier, causing liquid to penetrate into the brick and corrode the internal structure. It is easy to reduce its physical properties. Use in the future Cracked or broken in the middle.

\subsection{Pay attention to ceiling waterproof}

The cultural relic building has not used modern equipment such as air conditioners in the years of its existence. After the restoration, in order to improve the airtightness of the air- 
conditioned rooms, the patios, doors and windows closed with inertial thinking, and the continuous use of air-conditioning and fresh air equipment to control the temperature, not only caused waste of energy, but also In addition, in order to increase the air conditioning equipment, the original spatial layout and ceiling style of the building were modified to damage the authenticity of the cultural relics.

The enclosure structure of Lingnan building has a certain degree of air permeability. Its patio can allow direct sunlight to shine indoors, increase indoor air temperature, and use thermal pressure to form a chimney effect, natural ventilation and dampness; the north-south orientation of the building is conducive to wind pressure to organize the wind. Increase natural indoor ventilation. The above two points of building passive ventilation design have not been reflected in the restoration, and the current air-conditioning equipment not only has poor dehumidification capacity, resulting in water condensation on the ceiling, and the ceiling itself has not been fully waterproofed and mold-proof, not only cannot restore the original building The ventilation and dehumidification capacity of the building also self-defeatingly drives the historical reversing, which makes the indoor mildew phenomenon, and further affects the air quality and the quality of cultural relics, which is not conducive to the sustainability of cultural relics.

It is necessary to fully understand the intention of the ancients on the structure and function of the building, and avoid excessive interference of modern equipment on the cultural relics. If it is necessary to use modern equipment, a complete and timely evaluation standard and system should be established after the building is used, and the problems such as water accumulation and mold should be dealt with and corrected in time to avoid the problem from becoming out of control.

\subsection{Principles of brick repair}

Salty bricks should not be used in the restoration of cultural relics, which not only violates folk customs, but also is not conducive to the protection of cultural relics. Targeted repairs should be made to the damage of the blue brick in different situations: (1) When the damage of the blue brick is less than $2 \mathrm{~cm}$, the damaged part should be cleaned in time and the brick body should be kept. (2)When the damage of the blue brick is equal to $2 \mathrm{~cm}$, use old bricks to fill it, avoid using cement and other materials for filling. (3) When the damage of the blue brick is greater than $2 \mathrm{~cm}$, new blue bricks should be replaced. Avoid using acidified salty bricks. Try to use bricks with the same color, material and craftsmanship, and good quality. (4) The brick body should not be filled too much, just stop at the point. After the lime slurry is used to fill it and dry, it is washed with clean water as a whole, and then the brick joints are used to fill the joints. Finally, the wall is coated with clear limestone to make the brick body form a new protective film.

Bricks with better quality should meet the following conditions: (1)The brick surface is free of mold, dirt, and fading. (2) The brick body has small voids, compact material and sufficient materials. (3)The internal quality of the brick body is good, without water and corrosion, which can be distinguished by knocking the brick body, and the knocking sound is better. (4)The surface of the brick body is smooth, without roughness and impurities.

In addition, the maintenance of the brick body should be manually cleaned with soluble compounds. Cover the dust on the surface of the brick body with a wet towel, and then use tools such as toothbrushes, brushes, bamboo sticks, etc. to scrub with clean water, and then clean the wall with clean water. rinse. The brick wall should be maintained and maintained regularly to extend the life of the wall brick and enhance its quality. 


\subsection{Recommendations for wall desalination}

The use of injections to remove salt by the Chong Sai Pharmacy not only requires drilling holes in the brick wall and destroying the brick wall structure, but the effectiveness of the salt removal effect on the unique double-corner wall structure of Lingnan Architecture also needs to be verified. At present, there are many non-destructive desalination technologies that will not damage the structure of the brick wall, and the process is simple, and the effect has been confirmed, such as the paste desalination method, the humidity difference desalination method, and the mortar desalination method. The following is an analysis of the steps of the method.

\subsection{Pasting and removing salt}

Pasting desalination method is the use of compound chemical solutions to non-destructive desalination of brick walls [9], the specific steps are: (1) Prepare an aqueous solution of sodium hexametaphosphate (or potassium hexametaphosphate) with a content of $5 \%-15 \%$, Stir thoroughly under the condition of $20-50^{\circ} \mathrm{C}$, use towel or absorbent paper to fully wet the solution, and then apply it to the surface of masonry cultural relic wall to remove salt. (2) After desalting is completed, mix and stir dodecyltrimethoxysilane and ethanol at a ratio of 5:100 at $50-70^{\circ} \mathrm{C}$ to make a solution, and brush the solution to the wall to form a protective layer.

\subsection{Humidity difference desalination method}

The humidity difference desalination method uses the principle of osmotic pressure of air humidity for desalination. The specific steps are: (1)Detect and evaluate the actual situation of the water content and salinity of the brick body. (2)Adjust indoor air humidity and salinity so that the air humidity and salinity are the same as the humidity and salinity in the bricks. After a few days of rest, due to the principle of osmotic pressure, the brick body salt will be discharged naturally. (3) Clean the surface of the brick body with clean water, and repeat the above steps until the salt content of the brick wall reaches the desired level.

\subsection{Mortar desalination method}

The principle of the mortar desalination method is to use the mortar's salt adsorption characteristics to remove salt from the wall. The specific steps are: (1)Mix plant fibers (such as paper or grass tendons) and clay to prepare a neutral mortar solution. (2) Clean the wall with clean water. When the moisture is not dry, apply the mortar solution to the surface of the wall with a thickness of 10-20 mm. (3)Wait for 7-14 days, remove the dry mortar, clean the wall with clean water, and repeat the above steps until the salt content of the brick wall reaches the desired level.

\section{Summary}

The Chong Sai Pharmacy has important historical, social and cultural value. The restoration of its cultural relics will help fill in the archeological evidence of Sun Yat-sen's historical footprint in Macau, enhance the cultural connotation of Macau's city, enrich Macau's historic architectural attractions, and extend Macau's historical tourist routes Enhance the attractiveness of Macau as a tourist city, promote the spirit and culture of the Chinese nation, and promote national unity and harmony. However, in the restoration of cultural relics, 
attention should also be paid to the use of scientific and dialectical methods for restoration and restoration projects, retaining the original appearance of historical buildings, respecting the authenticity of the site and the memory of the residents, and retaining as much as possible for future archaeological research The historical materials in kind. At the same time, attention should be paid to the deficiencies in the restoration of the current status of cultural relics, and the restoration projects of cultural relics should be improved by analogy, increase the postuse evaluation system of the restoration of cultural relics, examine the causes of problems and study countermeasures, and summarize the technical texts. Macau is a similar area Provide reference and reference for the restoration work of cultural relics.

\section{References}

1. http://www.waou.com.mo/news_a/shownews_hary.php?lang=cn\&id=2516

2. http://www.macauzine.net/?action-viewnews-itemid-1089

3. Liu Xianbing, Culture Magazine 81 (2011)

4. Cai Peiling, Culture Magazine 81 (2011)

5. Lin Guangzhi, Cultural Magazine 81 (2011)

6. Chen Shurong, Sun Zhongshan, Chong Sai, Culture Magazine 81 (2011)

7. Sun Yat-sen, London's misfortune (Public Branch of China Social Sciences Press, Beijing, 2011)

8. Finding the starting point of Sun Yat-sen's success at No. 80 Cao Dui Street Ta Kung. Macao Special Edition (2017)

9. Wang Su, Huang Siping, Li Junlian, Zheng Jianguo, Zhang Wei, Qian Chunyu, Composite type desalination and protection material for masonry cultural relics and its preparation method and application. Chinese invention patent, patent number CN201510990645.9(2015) 